\title{
AUDITORÍA DE PREVENCIÓN TRIBUTARIA E INCIDENCIA EN EL PAGO DEL IMPUESTO GENERAL A LAS VENTAS EN EMPRESAS DE CALZADO
}

\author{
TAX PREVENTION AUDIT AND IMPACT ON THE PAYMENT OF THE GENERAL \\ TAX ON SALES IN FOOTWEAR COMPANIES
}

Greta Magaly Castillo del Águila Universidad Nacional de Trujillo Trujillo, Perú

ORCID: https://orcid.org/0000-0002-9892-8362 Correo electrónico: grecasticda87@hotmail.com

\section{RESUMEN}

Objetivo: Determinar en qué medida la aplicación de la auditoria de prevención tributaria incide en el pago del Impuesto General a las Ventas en empresas de calzado en Trujillo en el año 2017. Método: El tipo de investigación fue cuantitativa. Se trabajó con la información de tres empresas de calzado de la Asociación de Pequeños Industriales y Artesanos de Trujillo. Se aplicó la técnica de encuestas por cuestionario, análisis documental y entrevista a los contadores de cada empresa. Resultados: La auditoría de prevención tributaria en las empresas de calzado disminuyó en un $66,7 \%$ tanto en el incumplimiento tributario del Impuesto General a las Ventas; como en las fiscalizaciones de la Superintendencia Nacional de Aduanas y de Administración Tributaria del año 2017. Conclusión: La auditoría de prevención tributaria aplicada a empresas de calzado de Trujillo disminuyó de manera significativa el déficit de pago del Impuesto General a las Ventas del año 2017.

Palabras clave: Auditoría tributaria; impuestos; preventiva, infracciones.

\begin{abstract}
Objective: To determine to what extent the application of the tax preventive audit affected the General Sales Tax payment in footwear companies in Trujillo in 2017. Method: The investigation was quantitative, and it was developed with the information of three footwear companies from the Association of Small Industrialists and Artisans of Trujillo. The accountants of each company were subjected to the questionnaire survey technique, to the documentary analysis, and to a series of interview. Results: The tax preventive audit in footwear companies decreased by $66.7 \%$ in both, the tax non-compliance of the General Sales Tax, and the audits of the National Superintendency of Customs and Tax Administration in 2017. Conclusion: The preventive tax's audit applied to footwear companies in Trujillo, significantly reduced the payment deficit of the General Sales Tax for the year 2017.
\end{abstract}

Keywords: Tax audit; taxes; preventive; infraction.

( ) Los autores. Este artículo es publicado por la revista Quipukamayoc de la Facultad de Ciencias Contables, Universidad Nacional Mayor de San Marcos. Este es un artículo de acceso abierto, distribuido bajo los términos de la licencia Creative Commons Atribución 4.0 Internacional (CC BY 4.0) [https://creativecommons.org/licenses by/4.0/deed.es] que permite el uso, distribución y reproducción en cualquier medio, siempre que la obra original sea debidamente citada de su fuente original. 


\section{INTRODUCCIÓN}

Este trabajo muestra en qué medida la aplicación de la auditoría de prevención en materia tributaria repercute en el pago del Impuesto General a la Ventas (IGV) en la Asociación de Pequeños Industriales y Artesanos de Trujillo (en adelante APIAT) en el año 2017. Existe riesgo y peligro latente a que el profesional contable pueda incurrir en errores de índole tributario al llevar a cabo una contabilidad sencilla o compleja, lo que se puede evitar aplicando la mencionada forma de auditoría del IGV en cualquier compañía. Es preciso mencionar que los beneficios de este trabajo especializado no son conocidos ni promovidos en las empresas del sector calzado de la APIAT debido a la idea errada que tienen los empresarios de que no serán fiscalizados por la SUNAT; así también, por la confianza absoluta en el trabajo y opinión del contador, quien viene trabajando durante mucho tiempo.

Desde la perspectiva teórica, este estudio establece diversos tipos de proposiciones que se verán a continuación, los cuales permiten desarrollar juicios lógicos acerca de la relación que existe entre la auditoria tributaria de prevención del IGV y la disminución de infracciones tributarias por parte de las empresas del sector calzado que forman parte de la APIAT y, a su vez, la disminución de intervenciones de fiscalización tributaria de la SUNAT a estas empresas. Asimismo, los resultados de esta investigación pueden servir como fuente de información para trabajos similares, e incentivar las auditorías tributarias preventivas del IGV en empresas del mismo sector en nuestro país.

Al respecto, en una investigación realizada por Abanto (2017), el autor llegó a la conclusión de que la auditoría de prevención de impuestos tuvo una influencia positiva para determinar el IGV y el Impuesto a la Renta en la empresa materia de su investigación, puesto que la aplicación de dicho proceso disminuyó el pago de sanciones por multas más intereses moratorios al momento de ser fiscalizada por la SUNAT. Por otro lado, de acuerdo con lo indicado por Espinoza (2018), la puesta en marcha de la auditoría de prevención en impuestos, ayuda a detectar los errores tributarios de las empresas antes de enfrentarse a una fiscalización por parte de la SUNAT, y terminarla con éxito. Del mismo modo, Apaza (2017) afirma que la aplicación de la referida auditoría influye significativamente en la fiscalización tributaria de las empresas que formaron parte de su investigación. Así también, Mogollón (2017) concluye que, a través de la auditoría de prevención tributaria, se obtiene un ahorro fiscal que se puede medir gracias a esta herramienta, de modo que el dinero ahorrado permite mantener una buena rentabilidad en la empresa que la aplique.

Aliaga (2018), en su estudio, determinó que la aplicación de la auditoría de prevención tributaria trae como consecuencia la disminución de sanciones por tributos a cargo de la SUNAT, porque ya que permite subsanar los errores tributarios antes de alguna visita fiscalizadora de dicha entidad, y así minimizar el importe por pago de multas o determinación del impuesto que se fiscalice.

García (2017) demostró que la buena práctica de la auditoría de prevención en materia tributaria en las empresas ecuatorianas contribuyentes del Estado es necesaria para reducir la tasa de infracciones tributarias y, en consecuencia, aumentar la formalización de las empresas en el sentido de que la cultura tributaria mejorará para que las empresas existentes paguen impuestos de acuerdo con lo que verdaderamente obtienen como ganancia.

Ventura (2016), en su tesis de maestría en la que también se refiere al uso de la auditoría de prevención en cuestiones tributarias, concluye que los procesos contables de las empresas que formaron parte de su trabajo de investigación no cuentan con los requisitos para prevenir infracciones y sanciones administrativas. Esto debido a múltiples razones, siendo la más evidente la deficiencia de los contadores para la determinación de sus impuestos por la falta de dominio de las normas de índole tributaria de su país y de las Normas Internacionales de Auditoría, por lo que el autor recomienda capacitación constante al respecto para los profesionales de contabilidad.

Según indica León (2018), "la auditoría tributaria privada tiene una naturaleza eminentemente preventiva: la de evitar riesgos o que se produzcan contingencias tributarias" (p. 43). En otras palabras, la auditoría tributaria viene a ser la realización de un examen exhaustivo y especializado del adecuado cálculo de los impuestos que cada empresa está obligada a pagar de acuerdo con los parámetros que exija la ley, conforme al régimen tributario en el que se encuentre. Esto conlleva a que cada tipo de empresa cumpla con diversas obligaciones frente al fisco, las cuales pueden ser formales y/o sustanciales; y es justamente la auditoría tributaria, la que permite tener bajo control este aspecto de vital importancia en las empresas que aplican esta especialidad en su contabilidad.

\section{MATERIALES Y MÉTODOS}

El estudio tuvo un diseño cuantitativo y experimental. Para llevarlo a cabo se tuvo en cuenta como población a las empresas de calzado que conforman la APIAT, constituida por un total de 131 empresas de calzado, de acuerdo con la información brindada por la secretaría de la APIAT a través de un correo electrónico oficial. Se determinó una muestra de 3 empresas de calzado de las empresas que están asociadas a la APIAT. Para definir la muestra se llevó a cabo un muestreo por conveniencia, con una selección de muestra no probabilística, pues fueron seleccionados de 
acuerdo con la accesibilidad y proximidad de los sujetos para el investigador. La recolección de datos fue tomada en la oficina del contador y en el domicilio fiscal de cada una de las empresas del sector calzado integrantes de la APIAT que formaron parte de la muestra. Los procedimientos fueron aplicados al contador encargado de cada empresa, y fueron los siguientes: encuesta por cuestionario de preguntas con respuestas múltiples; encuesta por cuestionario de pregunta cerrada; análisis documental (consistentes en cédulas de auditoría) y entrevistas. Los datos obtenidos se procesaron con el software estadístico SPSS versión 24 , los resultados se presentan de acuerdo con los lineamientos de la estadística descriptiva, a través de tablas estadísticas con frecuencias absolutas y porcentuales, gráficos de barras, normalidad de los datos medidos antes y después, prueba de hipótesis para muestras relacionadas o pareadas y nivel de significancia 5\%. Para la comprobación de las hipótesis planteadas se aplicó la técnica analítica propuesta por Anderson, Sweeney y Williams (2008) "Prueba T-Student para muestras relacionadas o pareadas, pero siempre que los datos recopilados demuestren comportamiento de normalidad" (p. 410).

Del mismo modo, se menciona una serie de autores que apoyan la validez de este tipo de toma de muestra para realizar un trabajo de investigación (muestra pequeña):

La primera teoría indica que:

[...] para lograr establecer un grupo de resultados experimentales contenidos dentro de un intervalo de confianza determinado por el experimentador, es decir la garantía de que los datos son representativos, se debe establecer un número de muestra o réplicas de la actividad experimental que conlleve a la manipulación de datos de una alta fidelidad. El número de réplicas en la experimentación se establece mediante la utilización de herramientas estadísticas que minimicen la variabilidad de los datos observados. En ocasiones también se pueden incurrir al análisis de recursos disponibles en la experimentación tales como, material experimental (empresa), costo, número de experimentadores, tiempo. (Reyes, 2009, p. 8)

Fernandez, Trapero y Dominguez (2010) sostienen:

Que en cualquier experimento es, pues, muy importante tener en claro la magnitud correcta de la precisión deseada. No tiene sentido usar diez de tamaño de muestra o repeticiones para detectar una diferencia que se puede detectar con cuatro, ni tampoco realizar un experimento en el que el número de repeticiones sea insuficiente para detectar diferencias importantes, excepto ocasionalmente. (p.1)

Con respecto al procedimiento de auditoría preventiva aplicada, se procedió realizando un requerimiento de información contable direccionado a evaluar el IGV del año 2017 a los contadores de las empresas que conforman la muestra. Se aplicaron los siguientes exámenes que luego se plasmaron en cédulas de auditoría como sigue: (a) examen de los libros y registro de contabilidad; (b) examen de las declaraciones del pago del IGV; (c) verificación del débito fiscal a través de : exámenes de guías de remisión por ventas realizadas, de comprobantes de pago y notas de débito y crédito, cruce de comprobantes de pago y el registro de ventas, cruce de la base imponible registrada y la declarada; (d) verificación del crédito fiscal por medio de exámenes de comprobantes de pago y notas de débito y crédito, cruce de comprobantes de pago y el registro de compras; cálculo del crédito fiscal de operaciones gravadas y no gravadas; cruce del crédito fiscal registrado y el declarado, entre otros.

\section{RESULTADOS}

A continuación, se muestran los resultados obtenidos tras aplicar la auditoría de prevención tributaria del IGV en las empresas de calzado de la APIAT- durante el año 2017.

La figura 1 presenta a tres empresas del sector calzado que forman parte de la APIAT de las cuales, antes de la puesta en marcha de la auditoria de prevención en materia tributaria del IGV año 2017, el 33,3\% de ellos manifiesta que al día de hoy su empresa y/o negocio sí ha sido objeto de algún tipo de fiscalización del IGV, mientras que el $66,7 \%$ dijo que no. En cambio, después de la aplicación de la mencionada auditoría, estas mismas empresas (100,0\%) mencionaron al que al día de hoy su empresa y/o negocio no ha sido objeto de algún tipo de fiscalización del IGV.

Se evidencia la utilidad de la implementación de la auditoría de prevención de tributos en APIAT, pues ayuda a tener un mayor control de las oportunidades en las que cada empresa asociada fue fiscalizada y de qué manera se pueden subsanar errores para futuras fiscalizaciones, para el caso de estudio el impuesto sería el IGV y el periodo.

La figura 2 describe que las tres empresas del sector calzado de la APIAT tanto antes como después de aplicarles la auditoría de prevención tributaria del IGV, año 2017 dijeron en un $100,0 \%$ que para dicho periodo no le han detectado algún tipo de multa referida al IGV.

Se muestra un punto muy importante en la investigación, y es que la aplicación de la auditoría de prevención en materia tributaria del IGV en la APIAT para las unidades de negocio de calzado en el 2017 permitiría tener información 


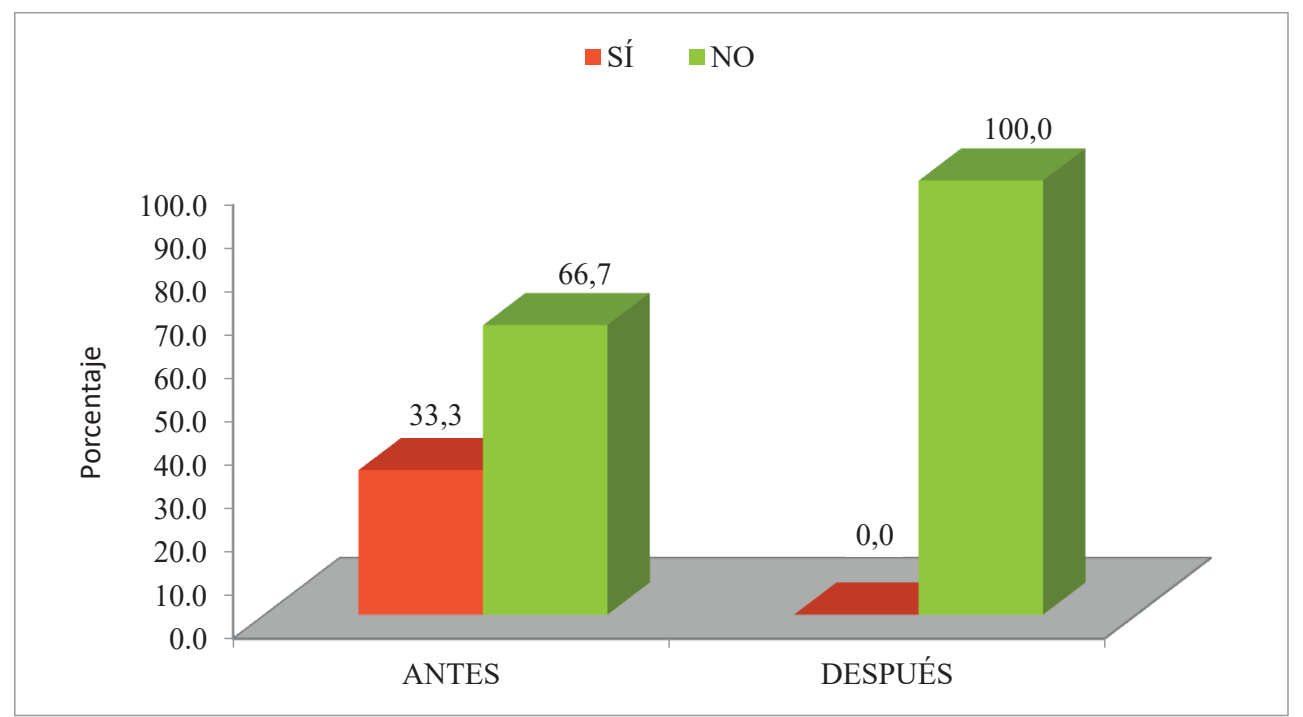

Figura 1. Empresas de calzado de la APIAT que han sido objeto de algún tipo de fiscalización del IGV por parte de SUNAT en el periodo 2017.

Fuente: Elaboración propia

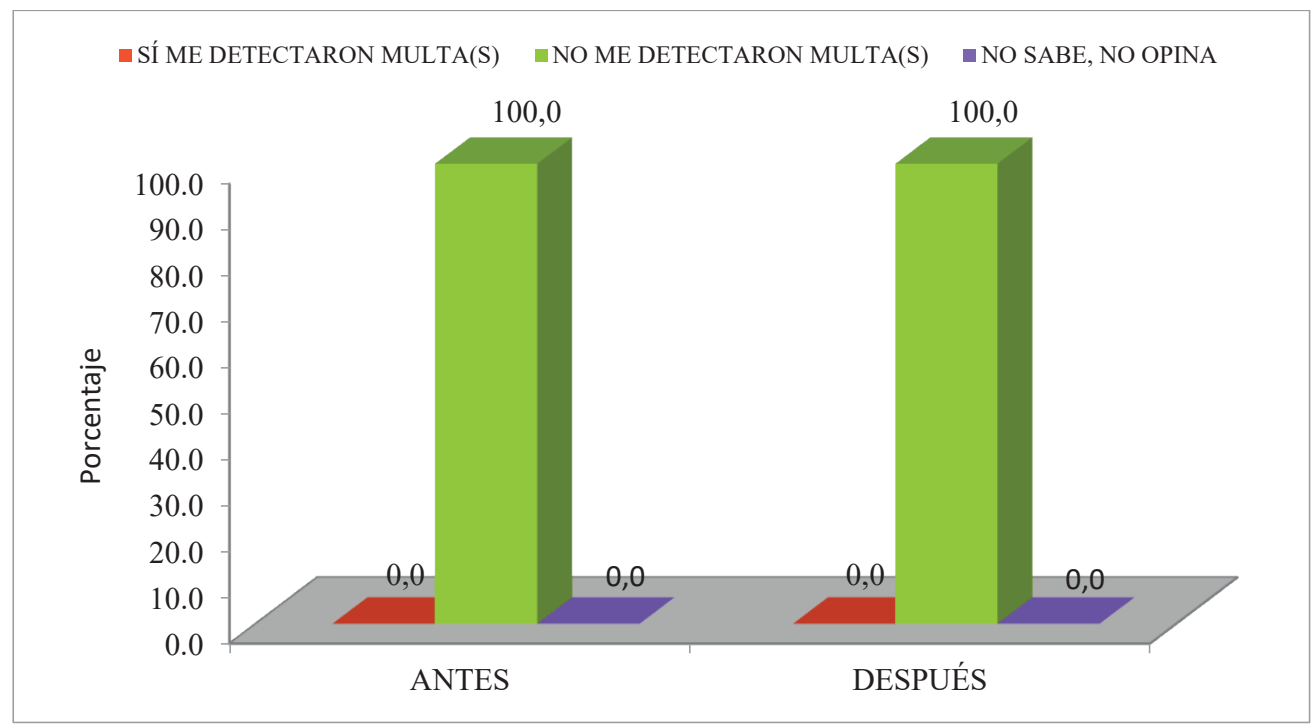

Figura 2. Empresas de calzado de la APIAT a las que se les detectaron multas después de una fiscalización del IGV por parte de SUNAT en el periodo 2017.

Fuente: Elaboración propia

precisa y correcta de cuánto es lo que se pagó (términos monetarios) al respecto de multas, de haber sido el caso, impuestas a cada empresa al afrontar cada fiscalización del IGV y, por ende, afrontarían con éxito un proceso de fiscalización. La SUNAT, al encontrar en esta situación a las empresas del mismo rubro, ya no gastaría los mismos recursos en procedimientos de fiscalización para estas empresas, pues vería el monto ínfimo de recaudación al término de cada proceso de fiscalización realizado.
La figura 3 muestra que al 100\% de las empresas del sector calzado encuestadas que forman parte de APIAT no se le ha detectado reparos tributarios ante algún tipo de fiscalización del IGV, ni antes ni después de la aplicación de la auditoria tributaria preventiva del IGV año 2017.

Se confirma de manera consecuente un panorama positivo para la aplicación de la auditoría de prevención en materia tributaria del IGV (2017) en las empresas de calzado de la 
APIAT, pues al no haber sido fiscalizados aún en el aludido tributo entonces no se les detectó reparos; por ello, estas empresas están a tiempo de detectarlos por su propia cuenta y con anticipación frente una visita fiscalizadora de la Administración Tributaria. Esto será posible aplicando la auditoría de prevención en materia tributaria del IGV, y poniendo en marcha las recomendaciones de auditoría que de ella se desprendan, pues es a nivel de reparos en donde finalmente se puede palpar la magnitud de ahorro que significa para la empresa la aplicación de la mencionada auditoría.

En la figura 4 se observa que el 33,3\% de las empresas mencionan que definitivamente sí es común la utilización de la auditoria de prevención tributaria del IGV, pero el $66,7 \%$

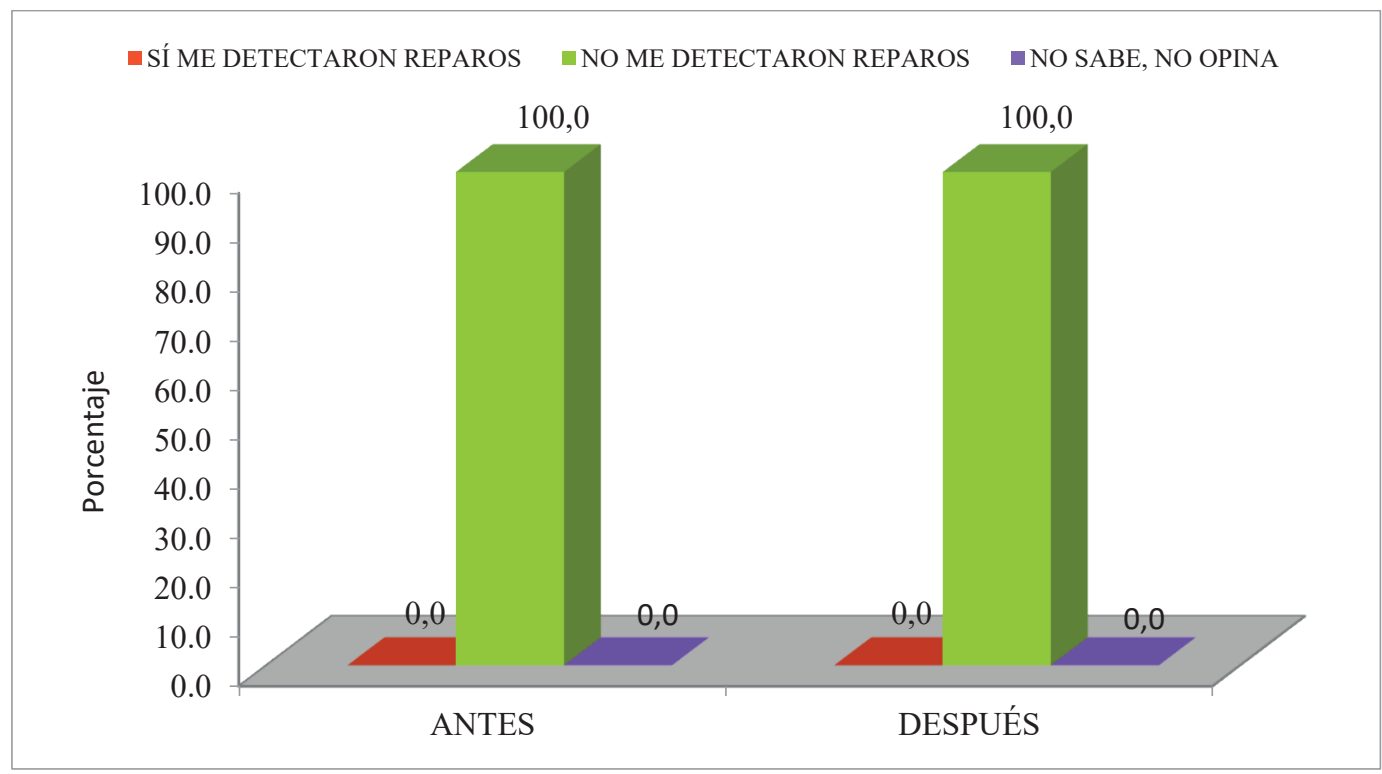

Figura 3. Empresas de calzado de la APIAT a las que les detectaron reparos tributarios después de una fiscalización del IGV por parte de SUNAT en el periodo 2017.

Fuente: Elaboración propia

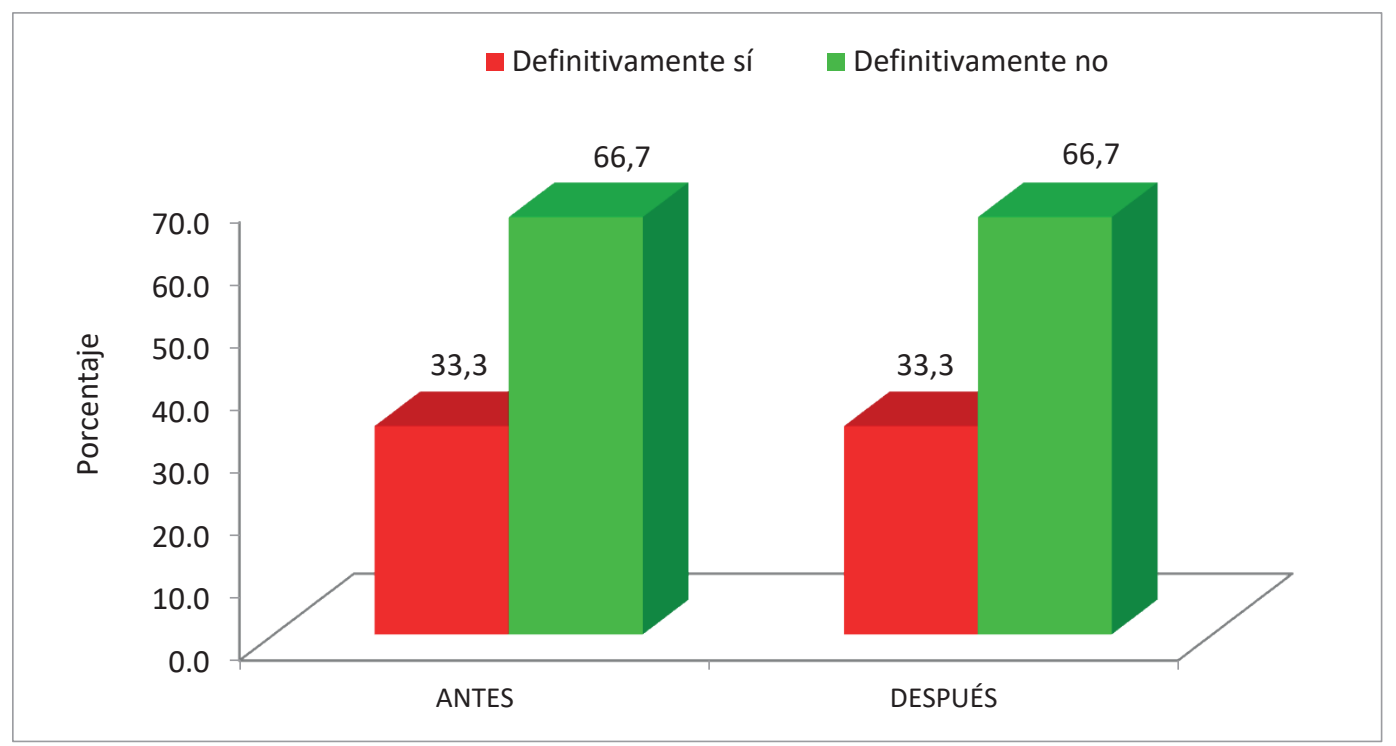

Figura 4. Grado de recurrencia de utilización de la auditoría tributaria preventiva del IGV en empresas de calzado de la APIAT en el año 2017.

Fuente: Elaboración propia 
mencionan lo contrario, ya que indican que definitivamente no es común su aplicación; esta respuesta se mantiene al realizar la encuesta tanto antes como después de dar a conocer los efectos de llevar a cabo la mencionada auditoría.

Observamos la situación actual respecto a la frecuencia de la aplicación de la auditoría tributaria de prevención en términos tributarios del IGV en las empresas de calzado de la APIAT, año 2017, y se muestra que una inminente mayoría del $66,7 \%$ indica que definitivamente no es frecuente la aplicación de esta útil herramienta y confirma los datos estadísticos mencionados al inicio de este trabajo mediante el planteamiento del problema. Los resultados de este trabajo de investigación, en el cual se recurrió al trabajo de campo de auditoría, son favorables, ya que es probable que este porcentaje pueda disminuir a lo largo del tiempo, y de esta manera, esta investigación pueda ser útil para los medianos, pequeños y micro- empresarios del sector calzado que forman parte de la APIAT.

La figura 5 muestra las tres empresas del sector calzado que manifiestan que antes de aplicarles la auditoria de prevención tributaria del IGV año 2017, el 66,7\% y 33,3\% probablemente no y definitivamente no cumplirán con las obligaciones tributarias del IGV. Sin embargo, después de recibir los resultados de la mencionada auditoria el 66,7\% y $33,3 \%$ de las empresas, definitivamente sí y probablemente sí cumplirán con las obligaciones tributarias del IGV en el referido periodo.
Los resultados obtenidos en la figura 5 son un indicador favorable, pues demuestran que al implementar las recomendaciones, que son consecuencia de aplicar la auditoría de prevención en materia tributaria del IGV en el año 2017 en empresas de calzado de la APIAT, el cumplimiento de obligaciones tributarias del IGV ante SUNAT ha aumentado en un $66,7 \%$ para dicho periodo; pues antes de recibir los resultados de la mencionada auditoría no se indicaba $(0,00 \%)$ que definitivamente sí contribuiría al cumplimiento de las obligaciones tributarias del IGV ante SUNAT en las empresas de calzado de la APIAT, y al conocer los resultados de la misma un $66,7 \%$ respondió que definitivamente sí contribuiría al cumplimiento de las obligaciones tributarias del IGV ante la SUNAT en el año 2017.

En la figura 6, se observa que antes de efectuar la auditoria de prevención en materia tributaria del IGV (2017), el $66,7 \%$ y el $33,3 \%$ de las empresas del sector calzado que forman parte de APIAT sostienen que la implementación de las recomendaciones de la mencionada auditoría, probablemente no y definitivamente no contribuirá a reducir la aplicación de fiscalizaciones de la administración tributaria, pero, después de que conocieron los efectos de haber aplicado la auditoría de prevención en asuntos tributarios del IGV para el año 2017, el 33,3\% y el 66,7\% de las empresas del sector calzado dijeron que definitivamente sí y probablemente sí contribuirá a reducir la aplicación de fiscalizaciones ante la SUNAT.

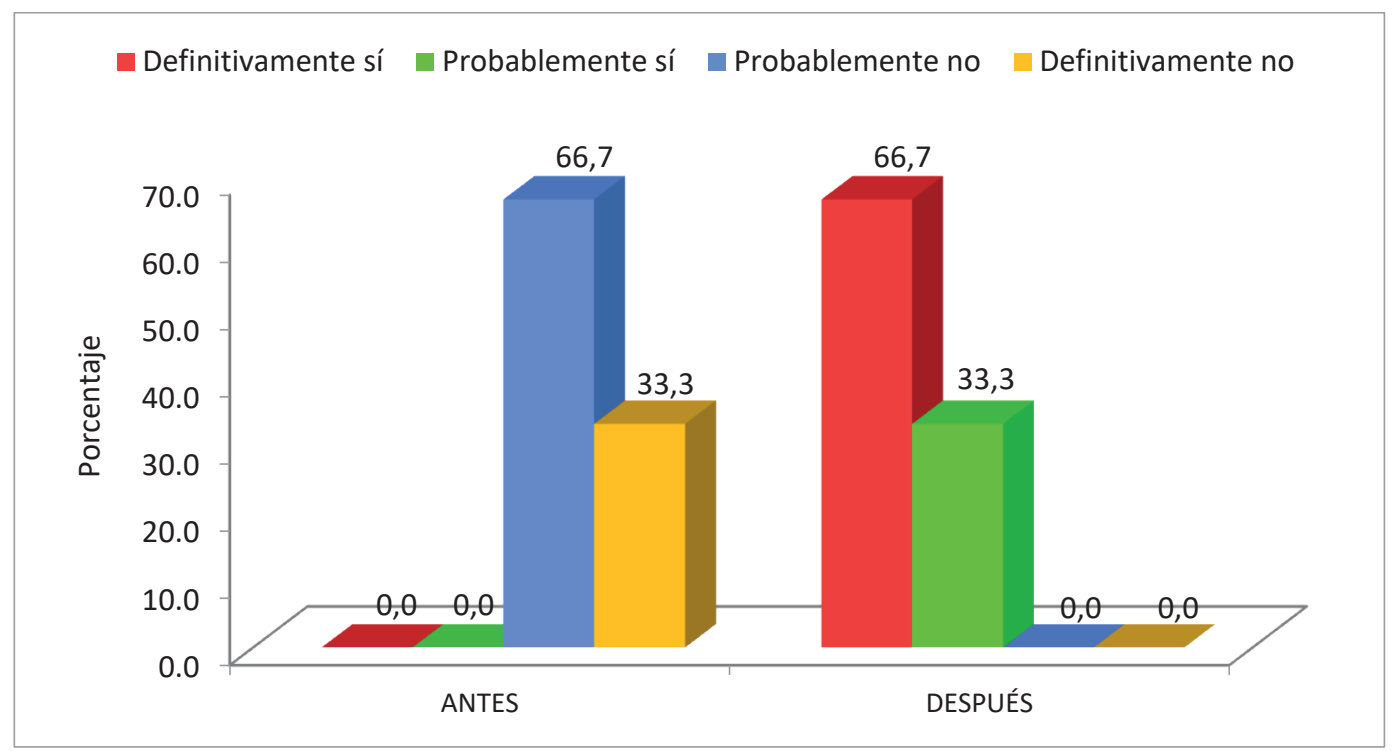

Figura 5. Contribución de la implementación de las recomendaciones de la auditoría tributaria preventiva del IGV al cumplimiento de las obligaciones tributarias del IGV ante la Administración Tributaria para el año 2017 en empresas de calzado de la APIAT.

Fuente: Elaboración propia 
En la categoría denominada probablemente sí de la figura 6 se muestra una variación positiva del $66,7 \%$ al aplicar la encuesta una vez conocidos los resultados de la auditoría de prevención tributaria del IGV con respecto a lo que indicaban las empresas antes de conocer dichos resultados ya figuraba con un $0.00 \%$, por tanto, la implementación de las recomendaciones de la auditoría de prevención tributaria del IGV en empresas del sector calzado que conforman la APIAT contribuirá a reducir en un 66,7\% la aplicación de procedimientos de fiscalización de la SUNAT en el año 2017 en dicha asociación.

En la figura 7, se obtuvo que antes de la aplicación de la auditoría tributaria preventiva del IGV en el año 2017, el 66,7\% y 33,3\% de las empresas de calzado de la APIAT expresaron que su aplicación probablemente

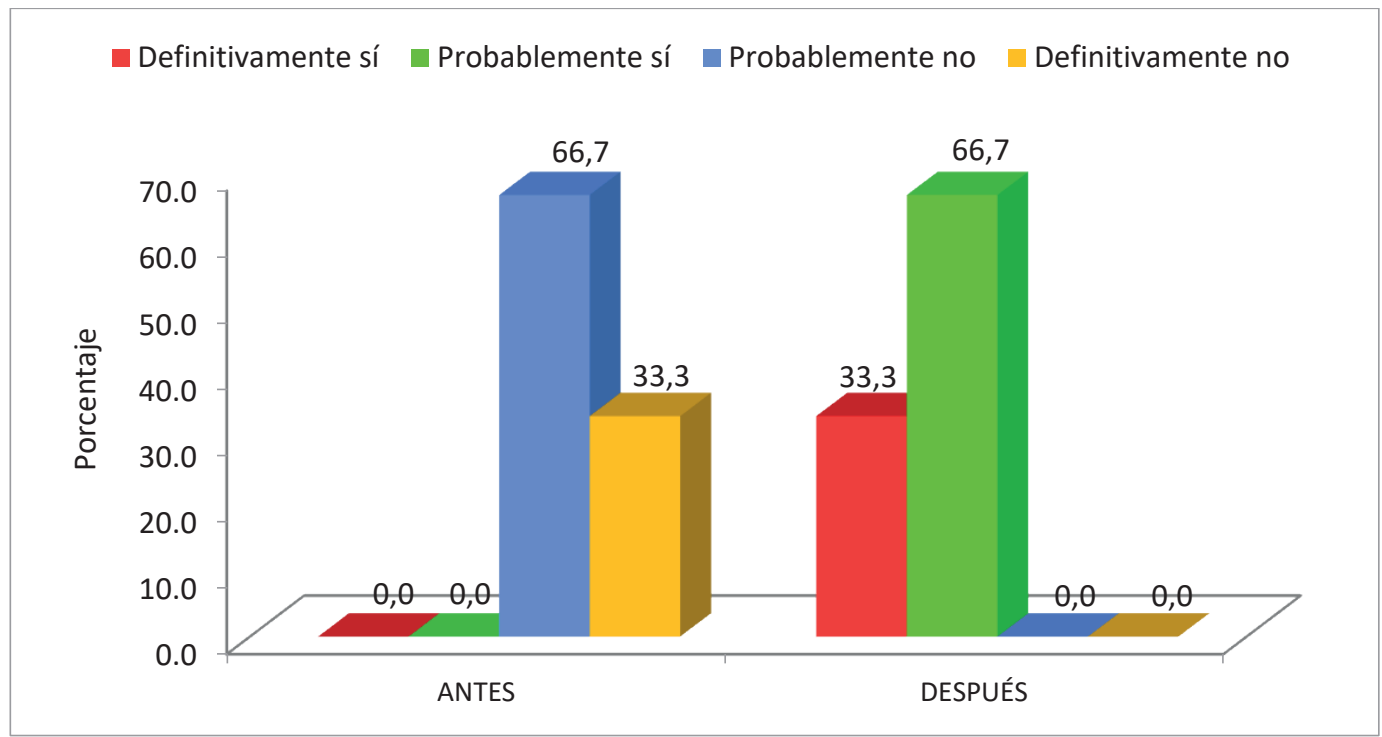

Figura 6. Contribución de la implementación de las recomendaciones de la auditoría tributaria preventiva del IGV en la reducción de aplicación de procedimientos de fiscalización de la Administración Tributaria para el año 2017 en las empresas de calzado de la APIAT.

Fuente: Elaboración propia

Definitivamente si Probablemente si Probablemente no Definitivamente no

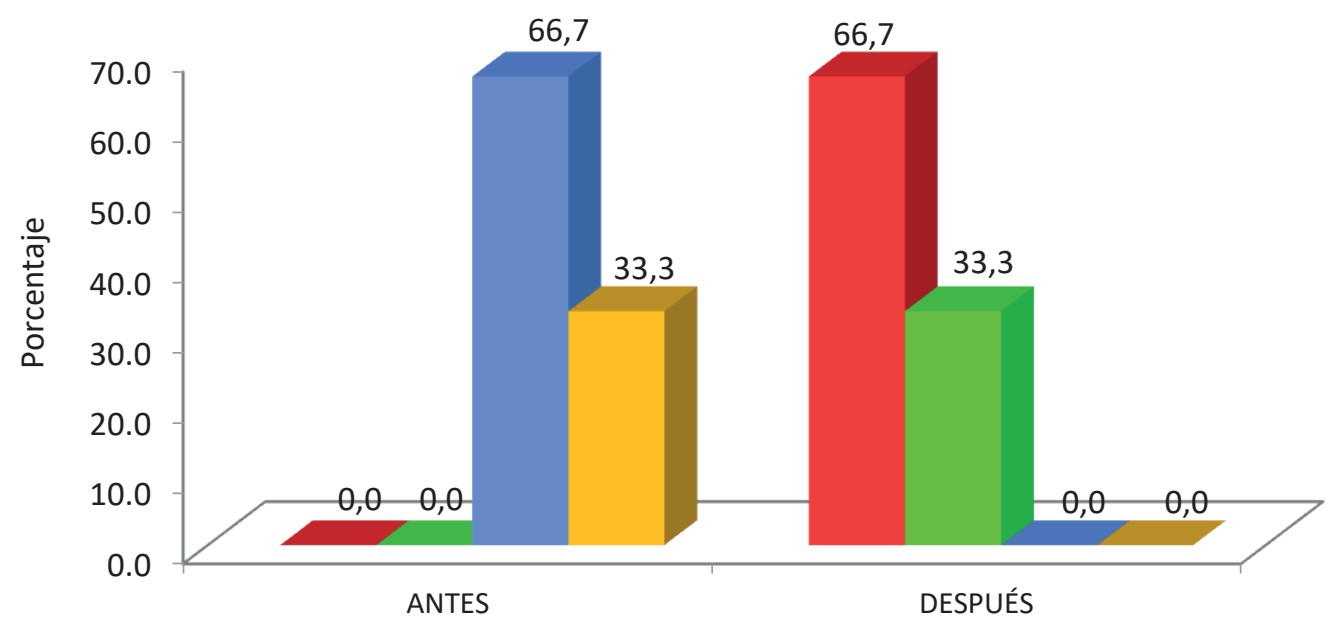

Figura 7. Contribución de la aplicación de la Auditoria Tributaria Preventiva en el pago del IGV en las empresas de calzado que conforman APIAT para el año 2017.

Fuente: Elaboración propia 
no y definitivamente no, incide en el pago del IGV y, después, cuando se les aplica la mencionada auditoría y conocen los resultados, el $66,7 \%$ y el $33,3 \%$ de dichas empresas sienten que definitivamente sí y probablemente sí el proceso incide en el pago del IGV.

En la categoría denominada definitivamente sí de la figura 7 se muestra una variación positiva del $66,7 \%$ con respecto a lo que indicaban las empresas antes de conocer los resultados de la mencionada auditoría que figuraba con un $0.00 \%$, por tanto, la puesta en marcha de la auditoría de prevención tributaria del IGV, en empresas del sector calzado que conforman la APIAT para el periodo 2017, incide positivamente en el pago del IGV en un $66,7 \%$.

Prueba de hipótesis

1. Hipótesis de Normalidad

$\mathrm{H}_{0}$ : El cumplimiento de las obligaciones tributarias del IGV, la aplicación de procedimientos de fiscalización y el pago del impuesto general a las ventas, antes y después se ajustan a una distribución normal.

$\mathrm{H}_{1}$ : El cumplimiento de las obligaciones tributarias del IGV, la aplicación de procedimientos de fiscalización y el pago del impuesto general a las ventas, antes y después no se ajustan a una distribución normal.

1.1. Nivel de significancia: $\alpha=0,05$

1.2. Valor significativo $(p-$ value $): p=0,363$ y $p=1,000$

1.3. Decisión: $\mathrm{p}>\alpha ; \mathrm{p}=0,363>0,05$ y $\mathrm{p}=1,000>0,05$

Se acepta la hipótesis nula, en consecuencia, el cumplimiento de las obligaciones tributarias del IGV, la aplicación de los procedimientos de fiscalización del IGV y el pago del impuesto general a las ventas, antes y después, se ajustan a una distribución normal.

2. Hipótesis general

$\mathrm{H}_{0}$ : La aplicación de la auditoría tributaria preventiva no incide de manera significativa en el pago del IGV en las empresas de calzado que conforman la Asociación de Pequeños Industriales y Artesanos de Trujillo (APIAT) para el año 2017.

$\mathrm{H}_{1}$ : La aplicación de la auditoría tributaria preventiva incide de manera significativa en el pago del IGV en las empresas de calzado que conforman la Asociación de Pequeños Industriales y Artesanos de Trujillo (APIAT) para el año 2017.

2.1. Nivel de significancia: $\alpha=0,05$

2.2. Valor significativo ( $\mathrm{p}-$ value): $\mathrm{p}=0,003$

\subsection{Decisión: $\mathrm{p}<\alpha ; \mathrm{p}=0,003<0,05$}

Se rechaza la hipótesis nula, en consecuencia, la aplicación de la auditoría tributaria preventiva incide de manera significativa en el pago del impuesto general a las ventas (IGV) en las empresas de calzado que forman parte de APIAT.

3. Hipótesis especifica (a)

$\mathrm{H}_{0}$ : La implementación de las recomendaciones de la auditoría tributaria preventiva del Impuesto General a las Ventas (IGV) en las empresas de calzado que forman parte de la APIAT no contribuye a disminuir la magnitud del incumplimiento de las obligaciones tributarias del IGV ante la Administración Tributaria para el año 2017.

$\mathrm{H}_{1}$ : La implementación de las recomendaciones de la auditoria tributaria preventiva del Impuesto General a las Ventas (IGV) en las empresas de calzado que forman parte de la APIAT contribuye a disminuir la magnitud del incumplimiento de las obligaciones tributarias del IGV ante la Administración Tributaria para el año 2017.

3.1. Nivel de significancia: $\alpha=0,05$

3.2. Valor significativo ( $\mathrm{p}-$ value): $\mathrm{p}=0,013$

3.3. Decisión: $\mathrm{p}<\alpha ; \mathrm{p}=0,013<0,05$

Se rechaza la hipótesis nula, en consecuencia, la aplicación de la implementación de las recomendaciones de la auditoria tributaria preventiva disminuyó la magnitud del incumplimiento de las obligaciones tributarias del IGV en las empresas de calzado que forman parte de la APIAT.

4. Hipótesis específica (b)

$\mathrm{H}_{0}$ : La implementación de las recomendaciones de la auditoría tributaria preventiva del Impuesto General a las Ventas (IGV) en las empresas de calzado que forman parte de la APIAT no contribuye a disminuir la magnitud de la aplicación de los procedimientos de fiscalización del IGV de la Administración Tributaria para el año 2017 en dicha asociación.

$\mathrm{H}_{1}$ : La implementación de las recomendaciones de la auditoría tributaria preventiva del Impuesto General a las Ventas (IGV) en las empresas de calzado que forman parte de la APIAT contribuye a disminuir la magnitud de la aplicación de los procedimientos de fiscalización del IGV de la Administración Tributaria para el año 2017 en dicha asociación.

4.1. Nivel de significancia: $\alpha=0,05$

4.2. Valor significativo ( $\mathrm{p}-$ value). $\mathrm{p}=0,005$ 


\subsection{Decisión: $\mathrm{p}<\alpha ; \mathrm{p}=0,005<0,05$}

Se rechaza la hipótesis nula, en consecuencia, la aplicación de la implementación de las recomendaciones de la auditoría tributaria preventiva disminuyó la magnitud de la aplicación de los procedimientos de fiscalización del IGV en las empresas de calzado que forman parte de la APIAT.

\section{DISCUSIÓN}

Los resultados en el trabajo de investigación coinciden con el estudio realizado por Abanto (2017), quien entre sus conclusiones indicó que la auditoría tributaria preventiva tuvo una influencia positiva en la determinación del IGV, y esto trajo como resultado evitar el pago de multas e intereses moratorios, en caso puedan ser fiscalizados por SUNAT. También coincide con lo indicado por Espinoza (2018), pues al haber aplicado la auditoría tributaria preventiva, se redujo el pago de sanciones tributarias incurridas de manera significativa, ya que las sanciones y omisiones se corrigieron voluntariamente y se acogieron a beneficios tributarios. En concordancia con lo concluido por Aliaga (2018), se demuestra que la auditoría tributaria preventiva ayuda a disminuir sanciones tributarias aplicadas por la SUNAT a los obligados tributarios y que contribuye a disminuir el importe de pago de sus multas, lo cual resulta muy beneficioso y, aunque la autora hace referencia a empresas del régimen del Impuesto a la Renta, en esta investigación se ha incluido como parte de la discusión porque en analogía terminaría siendo aplicable también para el IGV. En concordancia con García (2017), se demuestra que la aplicación de auditoría de prevención en materia tributaria en las empresas disminuye el margen de infracciones tributarias en general, para este trabajo de manera específica, viene a ser aplicable al IGV. Por tanto, se concluye que al poner en marcha las recomendaciones de la auditoría de prevención en materia tributaria del IGV en las empresas de calzado de la APIAT, disminuye la magnitud del incumplimiento de las obligaciones tributarias del IGV ante la SUNAT en el 2017; así también se reduce la aplicación de los procedimientos de fiscalización del IGV por parte de SUNAT en la APIAT, e incidió significativamente en el pago del IGV de las mencionadas empresas para el año 2017.

\section{REFERENCIAS}

Abanto, M. (2017). La auditoría tributaria preventiva del impuesto general a las ventas e impuessto a la renta en la empresa estructuras y montaje José Gálvez S.R.L. en el distrito de Cajamarca-2015 (Tesis de maestría). Universidad Nacional de Cajamarca. Recuperado de: http://repositorio.unc.edu.pe/handle/UNC/1076
Aliaga, L. (2018). La auditoría tributaria preventiva y las sanciones tributarias que aplica la SUNAT a los contribuyentes del régimen general del impuesto a la renta en el distrito de Huánuco, periodo 2016 (Tesis de maestría). Universidad de Huánuco. Recuperado de: http://repositorio.udh.edu.pe/bitstream/handle/123456789/942/ T047_70294850M.pdf? sequence $=1$ \&isAllowed $=\mathrm{y}$

Anderson, D., Sweeney, D. y Williams, T. (2008). Estadística para administración y economía. México, D.F.: Editorial Latinoamérica Cengage.

Apaza, J. (2017). Influencia de la auditoría preventiva en la fiscalización tributaria de las empresas constructoras de la ciudad de Juliaca año 2017 (Tesis de maestría). Universidad Andina Néstor Cáceres Valésquez, Juliaca. Recuperado de: http://repositorio.uancv.edu.pe/ bitstream/handle/UANCV/1524/T036_42412413.pd$\mathrm{f}$ ? sequence $=1$ \&isAllowed $=\mathrm{y}$

Espinoza, L. (2018). La auditoría tributaria preventiva y su influencia en el riesgo de una fiscalización tributaria en las empresas constructoras de la ciudad de Huánuco en el ejercicio fiscal 2015 (Tesis de maestría). Universidad de Huánuco. Recuperado de: http://repositorio.udh. edu.pe/handle/123456789/1297;jsessionid=70849FEFD8D5363FBB29C75A323C236F

Fernández, R., Trapero, A., y Domínguez, J. (2010). Experimentación en agricultura. Sevilla: Junta de Andalucía. Consejería de Agricultura y Pesca. Recuperado de:https://www.researchgate.net/publication/280558890_ Experimentacion_en_Agricultura

García, F. (2017). Propuesta para el fortalecimiento de la cultura tributaria a través de la auditoría tributaria (Tesis de maestría). Escuela Superior Politécnica del Litoral, Guayaquil. Recuperado de: https://www.dspace.espol.edu.ec/retrieve/101856/D-P13457.pdf

León, M (2018). Auditoría Tributaria Preventiva y Procedimiento de Fiscalización. Lima: Editorial Pacífico SAC.

Mogollón, X. (2017). La auditoria tributaria preventiva y su impacto en el riesgo tributario en la empresa Bilbao D y M EIRL periodo 2016 (Tesis de maestría). Universdad Católica Los Angeles Chimbote, Tumbes. Recuperado de: http://repositorio.uladech.edu.pe/handle/123456789/8539

Reyes, M. (2009). Aplicación del diseño experimental en el desarrollo de las prácticas internas, en el área de operaciones unitarias (Tesis de pregrado). Universidad San Carlos de Guatemala. Recuperado de: http://biblioteca.usac.edu.gt/tesis/08/08_1138_Q.pdf 
Ventura, J. (2016). Aplicación de procedimientos de auditaría tributaria preventiva a procesos contables de empresas Cafetaleras, Ocotal 2014 (Tesis de maestría). Universidad Nacional Autonoma de Nicaragua, Managua. Recuperado de: http://repositorio.unan.edu. ni/2695/1/17590.pdf 